\title{
Machine Reading Comprehension Using Structural Knowledge Graph-aware Network
}

\author{
Delai Qiu ${ }^{1}$, Yuanzhe Zhang ${ }^{2}$, Xinwei Feng ${ }^{4}$, Xiangwen Liao ${ }^{1}$, \\ Wenbin Jiang ${ }^{4}$, Yajuan Lyu ${ }^{4}$, Kang Liu ${ }^{2,3}$, Jun Zhao ${ }^{2,3}$ \\ ${ }^{1}$ College of Mathematics and Computer Science, Fuzhou University, Fuzhou, China \\ ${ }^{2}$ Institute of Automation, Chinese Academy of Sciences, Beijing, China \\ ${ }^{3}$ University of Chinese Academy of Sciences, Beijing, China \\ ${ }^{4}$ Baidu Inc., Beijing, China \\ noneqdl@gmail.com, \{yzzhang,kliu, jzhao\}@nlpr.ia.ac.cn \\ lxw@fzu.edu.cn, \{fengxinwei, jiangwenbin, lvyajuan\}@baidu.com
}

\begin{abstract}
Leveraging external knowledge is an emerging trend in machine comprehension task. Previous work usually utilizes knowledge graphs such as ConceptNet as external knowledge, and extracts triples from them to enhance the initial representation of the machine comprehension context. However, such method cannot capture the structural information in the knowledge graph. To this end, we propose a Structural Knowledge Graph-aware Network (SKG) model, constructing sub-graphs for entities in the machine comprehension context. Our method dynamically updates the representation of the knowledge according to the structural information of the constructed sub-graph. Experiments show that SKG achieves state-ofthe-art performance on the ReCoRD dataset.
\end{abstract}

\section{Introduction}

Machine reading comprehension (MRC) is an important subtask in natural language processing, which requires a system to read a given passage and answer questions about it. The ability of utilizing external knowledge is of great significance in an MRC system (Rajpurkar et al., 2016; Trischler et al., 2017). Latest large-scale datasets, e.g. ReCoRD (Zhang et al., 2018) specify that external knowledge is required to answer questions.

Many previous studies have introduced external knowledge in machine comprehension (Weissenborn, 2017; Mihaylov and Frank, 2018; Bauer et al., 2018). They often acquire external knowledge from structural knowledge graphs, such as ConceptNet (Speer et al., 2017) and Freebase (Tanon et al., 2016), in which knowledge is organized by triples like "(shortage, related_to, lack)" and "(need, related_to, lack)". However, most of them fail to make full use of the structural information in the knowledge graph, and use sequence modeling methods like recurrent neural networks (RNNs) to generate the representation of knowledge, rather than based on graph structure.

In this paper, we present a Structural Knowledge Graph-aware Network (SKG) model to leverage the structural information of external knowledge. The advantages of our proposed method are two folds: a) the constructed sub-graphs contain both MRC context and external knowledge nodes, reserving the knowledge structure; $b$ ) the graph neural network is capable of updating the nodes dynamically according to the structure of sub-graphs, instead of using external knowledge as pre-trained representations. Concretely, we first construct sub-graphs from external knowledge such as ConceptNet based on the context, and initialize the representation of nodes via knowledge graph embedding method (Yang et al., 2015). Then we employ graph attention networks to dynamically update the representation of nodes on sub-graphs. Finally, we utilize the final representation of nodes to augment the representation of context via gate mechanisms.

Our contributions can be summarized as:

- We present a simple but effective method to construct sub-graphs from a knowledge graph, which can reserve the structure of knowledge;

- Graph attention networks are employed to dynamically update the representation of knowledge based on sub-graph structure, which can make full advantage of structural information of external knowledge;

- Experiments demonstrate that SKG model is able to effectively leverage external knowledge in MRC task, and achieves state-of-theart performance on the ReCoRD dataset. 


\section{Related work}

External Knowledge Enhanced MRC Models There are several models that use knowledge for machine comprehension (Yang and Mitchel1, 2017; Mihaylov and Frank, 2018; Weissenborn, 2017; Bauer et al., 2018; Pan et al., 2019). Mihaylov and Frank (2018) relies on the ability of the attention mechanism to retrieve relevant pieces of knowledge, and Bauer et al. (2018) employs multihop commonsense paths to help multi-hop reasoning. They treat retrieved knowledge triples as sequences and use sequence modeling methods to compress the representation of knowledge, which are not based on graph structure. On the contrary, we organize knowledge as sub-graphs, then update the representation of nodes on sub-graphs with graph neural network.

\section{Graph Neural Netwoks}

Graph neural netwoks (Kipf and Welling, 2016; Schlichtkrull et al., 2018; Veličković et al., 2017) have been shown successful on many Natural Language Processing (NLP) tasks (Cao et al., 2018; Zhou et al., 2018; Song et al., 2018; Cao et al., 2019). They are good at dealing with graphstructured data. In (Cao et al., 2018; Song et al., 2018; Cao et al., 2019), Graph Convolutional Networks (GCNs) have been applied in multidocumnent machine comprehension for multi-hop reasoning question answering, but they consider only the internal structure information in the MRC context without incorporating external knowledge. To the best of our knowledge, our work is the first to study graph attention networks in machine comprehension with external knowledge.

\section{SKG Model}

The architecture of SKG is shown in Figure 1. It contains four modules: (1) Question and paragraph modeling module, which acquires the contextual representation of question and paragraph with BERT; (2) Knowledge sub-graph construction module, which retrieves sub-graphs from knowledge graph based on the context; (3) Graph attention module, which updates the representation of nodes on graph; (4) Output layer module, which is employed to generate the final answer.

\subsection{Question and Paragraph Modeling}

We first encode the tokens with BERT (Devlin et al., 2018). BERT has become one of the most successful natural language representation models

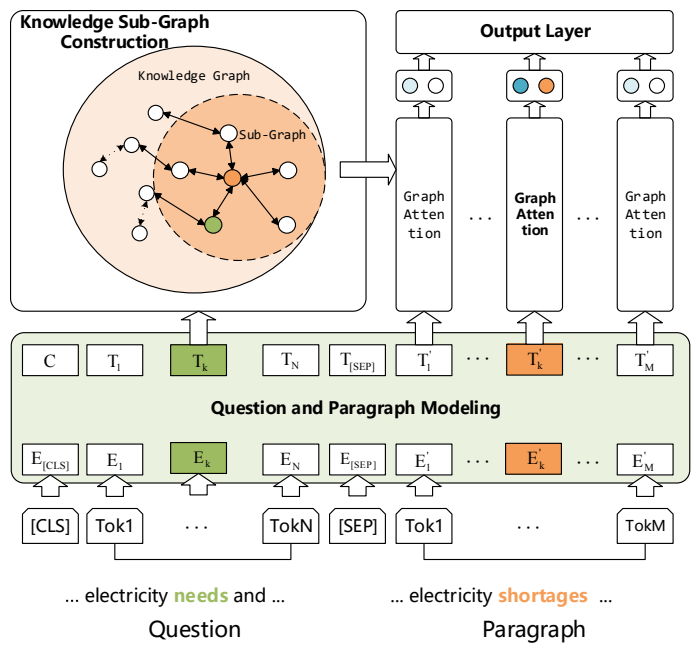

Figure 1: Framework of our SKG model.

on various NLP datasets, including SQuAD (Rajpurkar et al., 2016). BERT's model architecture is a multi-layer bidirectional Transformer (Vaswani et al., 2017) encoder, which is pre-trained on largescale corpus. We represent the input question and paragraph as a single packed sequence as follows:

\section{$[C L S]$ Question $[S E P]$ Paragraph $[S E P]$}

where $[C L S]$ is a specific classifier token and $[S E P]$ is a sentence separator which are defined in BERT. And we use BERT to generate the contextual representation of question and paragraph. The final hidden output from BERT for the $i^{t h}$ input token is denoted as $\boldsymbol{t}_{b_{i}} \in \mathbb{R}^{H}$, and $H$ is the output hidden size of BERT model.

\subsection{Knowledge Sub-Graph Construction}

We first use knowledge graph embedding approach to generate the initial representation of nodes and edges in terms of the whole knowledge graph.

We consider a knowledge triple in a knowledge graph as (head, relation, tail). For the $i^{\text {th }}$ token $t_{i}$ in paragraph, we retrieve all triples whose head or tail contains lemmas of the token. Take token "shortage" as an example, we retrieve triples like "(shortage, related_to, lack)". Then, we retrieve the neighbor triple of them, and reserve ones that contain lemmas of any token of the question. Thus we can acquire triples like "(need, related_to, lack)". We reorganize these triples into a sub-graph via connecting identical entities and reserving the relations as edges in these triples. So 
we can construct a simple sub-graph like "(shortage, related_to, lack, related_to, need)”, where "lack" is the identical entity. The sub-graph can be denoted as $g_{i}$ and the nodes and edges of it are initiated by the embeddings above.

\subsection{Graph Attention}

Our graph attention network is designed to update the representation of the nodes in a constructed sub-graph, which is inspired by (Veličković et al., 2017; Zhou et al., 2018). For the $i^{\text {th }}$ token $t_{i}$ in paragraph, its sub-graph is $g_{i}=\left\{n_{1}, n_{2}, . ., n_{k}\right\}$, where $k$ is the number of nodes. And $N_{j}$ is the set of the $j^{\text {th }}$ node neighbors.

The representation of nodes is updated $L$ times. At the $l^{t h}$ update, the updating rules are as follows, which are designed to model the interaction between the $j^{\text {th }}$ node and its neighbor nodes. In this way, the nodes are dynamically updated according to the structure of sub-graphs.

$$
\begin{gathered}
\boldsymbol{h}_{j}^{l+1}=\sum_{n=1}^{N_{j}} \alpha_{n} \boldsymbol{t}_{n}^{l} \\
\alpha_{n}=\frac{\exp \left(\beta_{n}\right)}{\sum_{j=1}^{N_{j}} \exp \left(\beta_{j}\right)} \\
\beta_{n}=\left(\mathbf{W}_{r}^{l} \boldsymbol{r}_{n}^{l}\right)^{\top} \tanh \left(\mathbf{W}_{h}^{l} \boldsymbol{h}_{n}^{l}+\mathbf{W}_{t}^{l} \boldsymbol{t}_{n}^{l}\right)
\end{gathered}
$$

where $\boldsymbol{h}_{j}^{l} \in \mathbb{R}^{d}$ is hidden state of the $j^{\text {th }}$ node, and its neighbor's hidden state is $\boldsymbol{t}_{n}^{l}$, and the hidden state of relation is $\boldsymbol{r}_{n}^{l}$, and $d$ is the hidden state dimension. $\mathbf{W}_{h}^{l}, \mathbf{W}_{t}^{l}$ and $\mathbf{W}_{r}^{l}$ are trainable weight matrices for the node, its neighbors and relations respectively. After $L$ updates, we can get the final hidden state of the central node as the final representation, which can be denoted as $\boldsymbol{t}_{k_{i}}$.

\subsection{Output Layer}

In the output layer, we combine this knowledge representation $\boldsymbol{t}_{k_{i}}$ with the textual representation $\boldsymbol{t}_{b_{i}}$ via a sigmoid gate, since external knowledge is not always necessary for reasoning.

$$
\begin{gathered}
w_{i}=\sigma\left(\left(W\left[\boldsymbol{t}_{b_{i}} ; \boldsymbol{t}_{k_{i}}\right]\right)\right) \\
\boldsymbol{t}_{i}^{\prime}=w_{i} \odot \boldsymbol{t}_{b_{i}}+\left(1-w_{i}\right) \odot \boldsymbol{t}_{k_{i}}
\end{gathered}
$$

We denote $T=\left\{\boldsymbol{t}^{\prime}{ }_{1}, \boldsymbol{t}^{\prime}{ }_{2}, \ldots, \boldsymbol{t}_{n}^{\prime}\right\}$ as the final representation, where $\boldsymbol{t}^{\prime}{ }_{i} \in \mathbb{R}^{H}$. And we study a start vector $S \in \mathbb{R}^{H}$ and an end vector $E \in$ $\mathbb{R}^{H}$ like (Devlin et al., 2018), which take $T$ as input. Then, the probability of the $i^{\text {th }}$ token being the start of the answer span is computed as a dot product between $T_{i}$ and $S$ followed by a softmax over all of the words in the paragraph:

$$
P_{i}^{s}=\frac{e^{S \cdot T_{i}}}{\sum_{j} e^{S \cdot T_{j}}}
$$

Let $P_{i}^{e}$ be the probability of the $i^{t h}$ token to be the end of an answer span, which can be calculated by the same above formula, and the maximum scoring span is used as the answer. The training objective is the loglikelihood of the correct start and end positions.

\section{Experiments}

\subsection{Dataset}

ReCoRD We report results on ReCoRD dataset (Zhang et al., 2018), a large-scale dataset for machine comprehension requiring external knowledge. There are 100,730, 10,000 and 10,000 examples in the training set, the development set and the test set respectively. The test set is not public, which needs to submit the model to the organization ${ }^{1}$ to get the results.

External Knowledge We consider two knowledge sources as our external knowledge: WordNet and ConceptNet. For WordNet, we use the preprocessed data provided by Bordes et at. (2013), which contains 151,442 triples with 40,943 synsets and 18 relations. For ConceptNet, we use the preprocessed data provided by Bauer et al. (2018), which contains 2,808,998 triples with 978,672 entities and 46 relations.

\subsection{Implementation Details}

Our model is implemented with pytorch ${ }^{2}$, and uses the framework ${ }^{3}$ for BERT model. We employ the open-source framework OpenKE (Han et al., 2018) to obtain the embedding of entities and relations with the BILINEAR model (Yang et al., 2015). The size of embedding of entities and relations is 100 . The update times $L$ of graph attention network is set to 5. We use Adam optimizer. The learning rate uses the linear schedule to decrease from 0.00003 to 0 .

\footnotetext{
${ }^{1}$ https://sheng-z.github.io/ReCoRD-explorer/

${ }^{2}$ https://github.com/pytorch/pytorch BERT
} 


\begin{tabular}{lllll}
\hline \multirow{2}{*}{ Model } & \multicolumn{2}{c}{ EM } & \multicolumn{2}{c}{ F1 } \\
\cline { 2 - 5 } & Dev & Test & Dev & Test \\
\hline QANet (Yu et al., 2018) & 35.38 & 36.51 & 36.75 & 37.79 \\
SAN (Liu et al., 2018) & 38.14 & 39.77 & 39.09 & 40.72 \\
DocQA w/o ELMo (Clark and Gardner, 2018) & 36.59 & 38.52 & 37.89 & 39.76 \\
DocQA w/ ELMo (Clark and Gardner, 2018) & 44.13 & 45.44 & 45.39 & 46.65 \\
SKG+BERT-Large(ours) & $\mathbf{7 0 . 9 4}$ & $\mathbf{7 2 . 2 4}$ & $\mathbf{7 1 . 5 5}$ & $\mathbf{7 2 . 7 8}$ \\
\hline
\end{tabular}

Table 1: The performance of different models on ReCoRD dataset.

\begin{tabular}{lll}
\hline Model & EM & F1 \\
\hline BERT-Base( 2018) & 54.03 & 55.99 \\
SKG+BERT-Base(ours) & 60.26 & 60.79 \\
BERT-Large( 2018) & 64.28 & 66.60 \\
SKG+BERT-Large(ours) & 70.94 & 71.55 \\
\hline
\end{tabular}

Table 2: The effectiveness of introducing external knowledge.

\begin{tabular}{lll}
\hline Model & EM & F1 \\
\hline MHPGM+NOIC(2018) & 28.36 & 29.29 \\
KG+LSTM+Bert-Base & 58.01 & 58.49 \\
SKG+BERT-Base(ours) & 60.26 & 60.79 \\
\hline
\end{tabular}

Table 3: The results of different ways of introducing external knowledge.

\subsection{Results and Analysis}

\section{Results on ReCoRD}

We choose several baselines: (1) QANet (Yu et al., 2018) is one of the top MRC models, which is different from many other MRC models due to the use of transformer (Vaswani et al., 2017). (2) SAN (Liu et al., 2018) is a top-rank MRC model, which employs a stochastic answer module. (3) DocQA (Clark and Gardner, 2018) is a strong baseline model, which consists of bidirectional attention flow and self-attention.

The results of different models are shown in Table 1. Our SKG+BERT-Large ${ }^{4}$ model achieves better performance than all previous published models, which is $26.13 \%$ higher in value than the state-of-art model DocQA with ELMo.

\section{The Effectiveness of External Knowledge}

Moreover, ablation experimental results on the dev $\operatorname{set}^{5}$ are given in Table 2. Once we re-

\footnotetext{
${ }^{4}$ Our first module is based on different BERT model sizes. BERT-Base contains $110 \mathrm{M}$ parameters and BERT-Large contains $340 \mathrm{M}$ parameters.

${ }^{5}$ Due to several weeks evaluation wait-time on the nonpublic test set, we just report results of our supplementary experiments on the dev set. In previous submissions, the re-
}

move the module incorporating external knowledge, the model degenerates into the fine-tuing BERT model, and the results show significant performance drop with $4.80 \%$ and $4.95 \%$ on different pre-trained model sizes respectively. The results demonstrate that our model can effectively utilize external knowledge.

\section{Different Ways to Use External Knowledge}

In the original paper of ReCoRD (Zhang et al., 2018), there is no research on existing models that use external knowledge to improve MRC task. So we study the recent model MHPGM+NOIC (Bauer et al., 2018), which utilizes multi-hop relational knowledge paths from ConceptNet. As shown in Table 3, our SKG model is more proper for introducing external knowledgse. In addition, to investigate the impact of the structural information on performance, we replace our sub-graph construction module with KG+LSTM, which retrieves knowledge triples without reconstructing the structure of them, and considers paths among them as sequences. We employ Long Short-Term Memory (LSTM) model to generate the representation of knowledge. As shown in Table 3, the performance drops $2.3 \%$ in F1, which means that the incorporation of structural information in the knowledge graph is able to make better use of external knowledge.

\section{Conclusion}

We propose SKG model for improving machine comprehension. Rather than treating triples from knowledge graph independently and separately, we construct sub-graphs from external knowledge. Then we generate the representation of knowledge with graph attention networks to improve the representation of context. Experimental results indicate that our model achieves the best performance in the challenging ReCoRD dataset.

sults in dev set are consistent with test set. 


\section{Acknowledgements}

This work was supported by National Key R\&D Program of China under Grant 2018YFB1005100, the National Natural Science Foundation of China (No.61533018, No.U1605251). This work was also supported by the Open Project of National Laboratory of Pattern Recognition at the Institute of Automation of the Chinese Academy of Sciences (201900041) and the independent research project of National Laboratory of Pattern Recognition.

\section{References}

Lisa Bauer, Yicheng Wang, and Mohit Bansal. 2018. Commonsense for generative multi-hop question answering tasks. In Proceedings of the 2018 Conference on Empirical Methods in Natural Language Processing, Brussels, Belgium, October 31 - November 4, 2018, pages 4220-4230.

Antoine Bordes, Nicolas Usunier, Alberto GarciaDuran, Jason Weston, and Oksana Yakhnenko. 2013. Translating embeddings for modeling multirelational data. In Advances in neural information processing systems, pages 2787-2795.

Nicola De Cao, Wilker Aziz, and Ivan Titov. 2018. Question answering by reasoning across documents with graph convolutional networks. CoRR, abs/1808.09920.

Yu Cao, Meng Fang, and Dacheng Tao. 2019. Bag: Bidirectional attention entity graph convolutional network for multi-hop reasoning question answering. arXiv preprint arXiv: 1904.04969.

Christopher Clark and Matt Gardner. 2018. Simple and effective multi-paragraph reading comprehension. In Proceedings of the 56th Annual Meeting of the Association for Computational Linguistics, ACL 2018, Melbourne, Australia, July 15-20, 2018, Volume 1: Long Papers, pages 845-855.

Jacob Devlin, Ming-Wei Chang, Kenton Lee, and Kristina Toutanova. 2018. BERT: pre-training of deep bidirectional transformers for language understanding. CoRR, abs/1810.04805.

Xu Han, Shulin Cao, Xin Lv, Yankai Lin, Zhiyuan Liu, Maosong Sun, and Juanzi Li. 2018. Openke: An open toolkit for knowledge embedding. In Proceedings of EMNLP, pages 139-144.

Thomas N Kipf and Max Welling. 2016. Semisupervised classification with graph convolutional networks. arXiv preprint arXiv:1609.02907.

Xiaodong Liu, Yelong Shen, Kevin Duh, and Jianfeng Gao. 2018. Stochastic answer networks for machine reading comprehension. In Proceedings of the 56th Annual Meeting of the Association for Computational Linguistics (Volume 1: Long Papers), pages 1694-1704.
Todor Mihaylov and Anette Frank. 2018. Knowledgeable reader: Enhancing cloze-style reading comprehension with external commonsense knowledge. In Proceedings of the 56th Annual Meeting of the Association for Computational Linguistics, ACL 2018, Melbourne, Australia, July 15-20, 2018, Volume 1: Long Papers, pages 821-832.

Xiaoman Pan, Kai Sun, Dian Yu, Heng Ji, and Dong Yu. 2019. Improving question answering with external knowledge.

Pranav Rajpurkar, Jian Zhang, Konstantin Lopyrev, and Percy Liang. 2016. Squad: 100,000+ questions for machine comprehension of text. In Proceedings of the 2016 Conference on Empirical Methods in Natural Language Processing, pages 2383-2392.

Michael Schlichtkrull, Thomas N Kipf, Peter Bloem, Rianne Van Den Berg, Ivan Titov, and Max Welling. 2018. Modeling relational data with graph convolutional networks. In European Semantic Web Conference, pages 593-607. Springer.

Linfeng Song, Zhiguo Wang, Mo Yu, Yue Zhang, Radu Florian, and Daniel Gildea. 2018. Exploring graph-structured passage representation for multihop reading comprehension with graph neural networks. CoRR, abs/1809.02040.

Robert Speer, Joshua Chin, and Catherine Havasi. 2017. Conceptnet 5.5: An open multilingual graph of general knowledge. In Proceedings of the ThirtyFirst AAAI Conference on Artificial Intelligence, February 4-9, 2017, San Francisco, California, USA., pages 4444-4451.

Thomas Pellissier Tanon, Denny Vrandecic, Sebastian Schaffert, Thomas Steiner, and Lydia Pintscher. 2016. From freebase to wikidata: The great migration. In Proceedings of the 25th International Conference on World Wide Web, WWW 2016, Montreal, Canada, April 11 - 15, 2016, pages 1419-1428.

Adam Trischler, Tong Wang, Xingdi Yuan, Justin Harris, Alessandro Sordoni, Philip Bachman, and Kaheer Suleman. 2017. Newsqa: A machine comprehension dataset. In Proceedings of the 2nd Workshop on Representation Learning for NLP, pages 191-200.

Ashish Vaswani, Noam Shazeer, Niki Parmar, Jakob Uszkoreit, Llion Jones, Aidan N Gomez, Łukasz Kaiser, and Illia Polosukhin. 2017. Attention is all you need. In Advances in neural information processing systems, pages 5998-6008.

Petar Veličković, Guillem Cucurull, Arantxa Casanova, Adriana Romero, Pietro Lio, and Yoshua Bengio. 2017. Graph attention networks. arXiv preprint arXiv:1710.10903.

Dirk Weissenborn. 2017. Reading twice for natural language understanding. CoRR, abs/1706.02596. 
Bishan Yang and Tom M. Mitchell. 2017. Leveraging knowledge bases in lstms for improving machine reading. In Proceedings of the 55th Annual Meeting of the Association for Computational Linguistics, ACL 2017, Vancouver, Canada, July 30 - August 4, Volume 1: Long Papers, pages 1436-1446.

Bishan Yang, Wen-tau Yih, Xiaodong He, Jianfeng Gao, and Li Deng. 2015. Embedding entities and relations for learning and inference in knowledge bases. In 3rd International Conference on Learning Representations, ICLR 2015, San Diego, CA, USA, May 7-9, 2015, Conference Track Proceedings.

Adams Wei Yu, David Dohan, Minh-Thang Luong, Rui Zhao, Kai Chen, Mohammad Norouzi, and Quoc V. Le. 2018. Qanet: Combining local convolution with global self-attention for reading comprehension. CoRR, abs/1804.09541.

Sheng Zhang, Xiaodong Liu, Jingjing Liu, Jianfeng Gao, Kevin Duh, and Benjamin Van Durme. 2018. Record: Bridging the gap between human and machine commonsense reading comprehension. CoRR, abs/1810.12885.

Hao Zhou, Tom Young, Minlie Huang, Haizhou Zhao, Jingfang Xu, and Xiaoyan Zhu. 2018. Commonsense knowledge aware conversation generation with graph attention. In Proceedings of the TwentySeventh International Joint Conference on Artificial Intelligence, IJCAI 2018, July 13-19, 2018, Stockholm, Sweden., pages 4623-4629. 